\title{
Clinical Presentation and Management of Neonatal Malaria: A Review
}

\author{
Kriti Mohan', B.J. Omar', and Rupa D. Singh ${ }^{3}$
}

${ }^{1}$ Assistant Professor, Department of Pediatrics, GSVM Medical College, \& Consultant at Mohan Children Hospital, Kanpur, India

${ }^{2}$ Associate Professor, Department of Microbiology, AlIMS, Rishikesh, India

${ }^{3}$ Associate Professor, Department of Pediatrics, GSVM Medical College, Kanpur, India

\begin{abstract}
The burden of neonatal malaria was thought rare in the past because of presence of protective factors such as maternal antimalarial antibodies, lactoferrin, secretary lgA in human milk and $\mathrm{HbF}$. In endemic areas for malaria, this group (neonates) needs to be more focused for such infection, as due to difficulty in diagnosis it may be representing only the tip of iceberg. In addition there is little information on the use of artimisinin-based combination therapy in neonates. This review aims to discuss all these aspects, focusing mainly on diagnosis and management of neonatal malaria. The final objective is to emphasize the need to establish management guideline of malaria in this neglected age group.
\end{abstract}

Keywords: Congenital malaria; Neonatal malaria; Artemisinin

\section{Introduction}

This review analyzes the issues regarding diagnosis and management of neonatal malaria with an aim to provide physicians, clear and concise information about the diagnosis and to emphasize the need to establish management guideline of neonatal malaria in the clinical practice. Malaria contributes significantly to perinatal disease burden in terms of pregnancy loss, prematurity and intra-uterine growth retardation (IUGR) [1-3]. Despite these well-documented indirect effects of malaria to the fetus and newborn, the direct burden of neonatal malaria infection in terms of prevalence and outcome is not well described in malaria endemic areas. Studies published so far have documented contradictory levels of this burden [4-7]. Most of these studies were conducted in large referral or teaching hospitals, and the results are difficult to generalize $[4-6,8,9]$. Furthermore, many studies lack details of internal and external laboratory quality control procedures, parasite densities and examine a small sample size of neonates with malaria [6-11] and thus these limitations further complicate the interpretation and application of the findings. Neonates have different clinical manifestations $[12,13]$ and lower parasite densities [14] than older children. Nevertheless, even low density (1500 parasites/ micro ml) in infants can result in anemia if left untreated [15], and may rapidly progress to become life threatening [16].

Neonatal malaria is considered a rare occurrence due to the protective effect of maternal immunity after birth. Maternal immunoglobulin $G$ ( $\operatorname{Ig} G$ ) antibodies are acquired by the fetus via the placenta in utero [17]. Although there is only limited evidence suggesting a protective role of these passively acquired antibodies against malaria parasites [18]. Ig G levels are thought to decrease variably during the first year of life [19]. Neonates may also be protected through factors that inhibit parasite growth, such as lactoferrin [which binds iron] and secretory IgA found in breast milk and in maternal and infant sera [20]. Parasite replication depends on the metabolic substrate para-aminobenzoic acid (PABA), which is present only in low levels in breast milk [17]. Fetal hemoglobin (Hb F), present in high concentration at birth [17] can inhibit parasite development [21] and can protect infant in the first few months of life. The P. falciparum Erythrocyte Membrane Protein-1 (PfEMP-1) mediates cytoadhearence of infected red blood cells to the endothelial cells lining the blood vessels. $\mathrm{HbF}$ and maternal IgG act in coordination to impair the cytoadherence of parasitized erythrocytes in the first few months of life by altering PfEMP-1 display on HbF RBCs, and by binding PfEMP-1 and preventing sequestration of parasitized RBCs, respectively [22]. $\mathrm{Hb}$ F levels decline after a peak level at 6 weeks of age [17], and as both IgG and $\mathrm{HbF}$ disappear from circulation, making infants more susceptible to malaria infection [22]. In countries with high malarial burden, the presentation of congenital malaria is relatively early (Mozambique and Benin from South Africa) [23,24]. The reasons are unclear however it can be related to prenatal fetal priming [25]. As clinical signs of neonatal malaria may be indistinguishable from that of neonatal sepsis, it has been suggested that screening of malaria parasite be included as a part of routine investigation in a neonate with fever [26]. Sometimes lack of high index of suspicion for neonatal malaria has unwittingly increased the duration of hospital stay of neonates and has also contributed to high morbidity and mortality. The burden of malaria in nonendemic countries had led to a suggestion by workers that neonatal malaria should be considered in all newborns born to mothers who had travelled to endemic areas [27]. Some reduction in malaria risks in neonates can probably be achieved by improving malaria control in late pregnancy and reduction in morbidity and mortality may be achieved by effective management of malaria in neonates.

\section{Burden of Neonatal Malaria}

Malaria in neonates is classified according to the time of acquiring infection. Congenital malaria, defined as asexual parasites detected in cord blood or peripheral blood during the first week of life [5], is due to transmission from the mother through placenta just before or during delivery [28]. While acquired neonatal malaria, which can occur within first 28 days of life, is due to an infective mosquito bite after birth [28]. Differentiating between congenital and acquired malaria can be difficult, especially in areas of intense malaria transmission [29].Various studies across Africa have demonstrated that $7-10 \%$ of newborns may have malaria parasites in their cord blood [15].

*Corresponding author: Dr. Kriti Mohan, G.S.V.M. Medical College, Kanpur, Uttar Pradesh, India; Tel: +919415557348; E-mail: kriti.mohan@rediffmail.com

Received June 24, 2014; Accepted October 16, 2014; Published October 25 2014

Citation: Mohan K, Omar BJ, Singh RD (2014) Clinical Presentation and Management of Neonatal Malaria: A Review. Malar Chemoth Cont Elimination 3: 126. doi: $10.4172 / 2090-2778.1000126$

Copyright: ( $) 2014$ Mohan K, et al. This is an open-access article distributed under the terms of the Creative Commons Attribution License, which permits unrestricted use, distribution, and reproduction in any medium, provided the original author and source are credited. 
Most literature on pediatric malaria originates from Africa, a hyperendemic region where it is the single largest killer of children between the 28 days to 4 years of age [30]. Although India is the largest contributor to the disease in the Southeast Asia Region, it is a low risk nation for mortality due to malaria [31]. Being a low endemic region, the median age of presentation tends to be later ( 8 years) in contrast to the mean age at 26 months reported from Africa [32]. In recent year, workers from endemic areas reported the incidence of neonatal malaria to be $58.5 \%[4,33]$. Studies examining the incidence of parasitemia in infant born in endemic region have shown rates varying from $0-33 \%$ [34,35]. A study from central India has shown a lower incidence [29/1000 live births] of congenital malaria. Low incidence of congenital malaria in this study could be because they may not have performed histopathological examination of placenta, which is a more reliable method of assessing placental infection than obtaining blood smear alone [36]. A study from Nigeria had identified a prevalence of $24.8 \%$ of acquired neonatal malaria and $17.4 \%$ of congenital malaria [37]. The occurrence of infection in utero is also reflected by the prevalence of splenomegaly at one month of age, which can be as high as $80 \%$, indicating an early development of a splenic response to the infection $[14,38]$. The marked difference in prevalence of acquired neonatal or congenital parasitemia is difficult to explain. Plausible explanations include statistical epidemiological differences and operational factors. In several recent studies, the routine slide reading for malaria was shown to have very poor positive predictive value when compared to expert microscopy $[39,40]$. In one centre, only $13 \%$ of adults with diagnosis of cerebral malaria actually had parasitemia on their blood film [41]. These data suggests that over-diagnosis of clinical malaria may be widespread in India and therefore may lead to under-treatment of other life threatening conditions. However differences in transmission dynamics, small sample sizes and lack of details on quality control and sample selection procedures make the interpretation of these findings difficult [5,42]. In countries with high malaria burden like South Africa it is usual to find some degree of parasitaemia in newborn with sepsis. So it becomes difficult to assign parasitaemia as a cause of ill health in these newborns. In addition one study suggests that septic neonates and those with suspected sepsis are likely to have parasitaemia [4].

\section{Clinical Sign and Symptoms and Malaria Diagnosis in Newborns}

In endemic areas it is often difficult to distinguish congenital from acquired malaria that develops 3 to 4 weeks after birth [43]. Normally symptoms of congenital malaria occurs 10 to 30 days post-partum, [44] which is the estimated half-life of maternal antimalarial IgG in the infant [45]. Malaria in the first few months of life can simulate infants presenting with intrauterine infections such as toxoplasmosis, rubella, syphilis, neonatal hepatitis and also those with septicemia. All of which have an important symptom complex of fever, jaundice, hepatosplenomegaly and anemia. Fever is the characteristic manifestation of neonatal malaria [46]. Several Indian studies [47-49] on neonatal malaria have reported fever (90$100 \%)$, hepatosplenomegaly $(70-80 \%)$, refusal to feed (40-70\%), loose motions (23-40\%) and respiratory distress (20\%) [47-50]. BR Thapa et al also reported intravascular hemolysis as a rare manifestation in one case of neonatal malaria [51]. Hence any baby in the first few months of life presenting from endemic areas with fever, anemia, and hepatosplenomegaly or other features like bronchopneumonia or septicemia should always be investigated for malaria.

Some antenatal and perinatal factors also play role in occurrence of congenital malaria. A study from Nigeria suggested that all febrile neonates should be evaluated for malaria especially if there is history of prolonged labor or prolonged length of rupture of membrane [52]. Malaria infection is more frequent and serious during the first pregnancy, as is the occurrence of congenital malaria but reasons are unclear [53] M. Singh et al mentioned blood film of patient's mother is usually negative for parasite in congenital malaria [54] In some cases there was no evidence of an active maternal infection or the parasite genotype was different to those found in the newborn. This may be explained on the basis that malaria parasite was transferred to the newborn followed by its clearance from maternal and placental blood $[15,55,56]$. In a study from South Africa, maternal malarial infection was associated with a low birth weight of baby [57]. However in a study from central India, no significant correlation was found between maternal malarial infection and its effect on either gestational weight or maturity [36].The plausible explanation of these contradictions could be explained on the basis of species of malaria parasite. In the former study it refers mostly to P. falciparum causing mostly severe malaria while in the later study it refers mainly to $P$. vivax causing malaria with milder forms usually.

In India cases of neonatal malaria was detected accidentally which are routinely investigated for other illnesses because they were not suspected having malaria [58]. So the dilemma of distinguishing the neonate with neonatal malaria alone versus neonatal sepsis or both existing does not seem to be easily resolved by the use of clinical features alone.The laboratory diagnosis of parasitemia in neonates require special attention in the preparation of Giemsa stain and buffer solution used, as well as in the technical skill involved in malaria microscopy because parasite densities are low. The sensitivity of detection of neonatal parasitemia may be better enhanced by more modern techniques of assay utilizing Polymerase Chain Reaction (PCR) for malaria nucleotide protein [e.g. MSP1 or MSP2] detection [59]. In many resources-poor countries like India (in malaria endemic zones) these latter techniques are not routinely feasible. The need to build capacity in malaria microscopy in these areas is therefore crucial. In areas with limited resources, the capacity to diagnose malaria in newborn may be limited $[60,61]$ and any issues with quality or accuracy of diagnostic technique may result in the diagnosis of malaria being missed [57]. Rapid diagnostic test may have significant diagnostic utility in neonatal malaria in which low parasetemia causes diagnostic difficulty through microscopy by non-experienced person. Emphasis should be placed on developing the capacity for rapid and reliable laboratory services with adequate quality control. Neonatal mortality contributes about forty percent to all child death in malaria endemic areas [62] and most of these death and deliveries occur outside health facilities so the proportion of these deaths that are malaria related remains undetermined and unrecorded $[23,63,64]$.

\section{Pharmacokinetics of Anti-Malarials in Newborns}

Due to immaturity and dynamic developmental changes experienced by newborns, the pharmacokinetic profiles of anti-malarial drugs may be different than in older children, and age dependent dose adjustment may be necessary, for example gastric emptying is slower in neonates and only comparable with adults after six month of age. Absorption of drugs is also affected by intestinal motor activity and villous formation, both of which mature by week 20 [65]. Activity of such metabolizing enzymes is typically low at birth and rapidly develops over the first 1-2 years of life [66,67].

\section{Current Recommendations for Treating Newborns}

Neonatal age group was excluded from clinical trials and WHO 
treatment guideline is not formulated for malarial infection in this age group. Thus, anti-malarials recommended are frequently used off-label, based on the recommended $\mathrm{mg} / \mathrm{kg}$ dosing schedule for older children [7].

\section{Issues with Currently Available Anti-Malarials}

There is little information on the use of artemisinin based combination therapy [ACT] in newborns, to the extent that many of them carry label restrictions for this age group [66]. Newborn with malaria should receive appropriate treatment with proper dosing schedule. Among the recommended ACT, excluding the combinations containing sulphadoxin-pyramethamine that is not recommended during the first 6 weeks of life [68], there is no evidence of specific serious toxicity [66]. However additional efforts should be made to establish their safety profile.

ACT offers greater efficacy and tolerability compared with quinine, which is often used in neonate with clinical malaria. In one study from Nigeria they used amodiaquine successfully in neonates [33]. In another study from Nigeria they treated uncomplicated neonatal malaria with chloroquine and sulphadoxin-pyramethamine as a first and second line respectively [69]. Furthermore, the use of a unified first-line therapy could offer benefits for healthcare providers with similar antimalarial use for the whole population, as well as logistical benefits in terms of procurement and distribution. The safety profile of these combination therapies in newborn is yet to be established

\section{Prevention of Neonatal Malaria}

More emphasis should be placed on interventions to reduce the burden of malaria in pregnancy. WHO has developed guidelines for control of malaria in pregnancy for Sub Saharan Africa which consists of prompt and effective case management of malaria illnesses combining with prevention of infection with insecticide treated nets and intermittent preventive treatment in pregnancy [70]. Following these broad guidelines, Nigeria has recently developed its own guideline policy for treatment of malaria in pregnancy with the use of intermittent preventive therapy using sulphadoxin-pyramethamine and insecticide treated bed nets [71]. However the implementation of these interventions will require concerted efforts to achieve effective control of maternal parasetemia and placental malaria in other endemic countries. The candidate malaria vaccine RTS, S/AS01 is of no use because it is ineffective in neonatal period because of presence of maternal antibodies and recommended to administer at 6-12 weeks of age.

\section{Future Clinical Studies}

There are several ongoing or planned clinical studies in other malaria prevalent countries like Africa aiming to obtain more accurate estimates of the malaria burden and morbidity as well as establish safety and efficacy of currently available ACT in newborns living under different endemic conditions. Future studies are also required to asses newborn iron status in relation to subsequent malaria risk, and relate findings to the occurrence of placental malaria.

Need of the day is that such type of studies must be carried out in other malaria-endemic countries also, so that knowledge gained through such studies may assist in guiding the development of evidence based treatment guidelines for this population in these countries.

\section{Conclusions}

Neonatal malaria is uncommon in newborns. This resulted in the paucity of information currently available and the lack of evidence based treatment guidelines in this population. Many children are dying before malaria is diagnosed. In resource-constrained settings, diagnostic techniques may not be available, or malaria may not be recognized due to overlapping symptoms with other neonatal illnesses such as sepsis. Malaria in newborns is not a rare occurrence in endemic areas and its burden may be underestimated. Therefore, it is necessary to collect reliable data on the malaria burden in this population. Awareness by health professionals should increase so that any newborn brought to a health facility in a malaria-endemic area with an unexplained fever or suspected sepsis should be systematically screened for malaria. Current policy for malaria diagnosis dictates that all fevers in all age groups and settings should be tested for malaria before treatment is initiated especially in endemic areas. ACT is being used off-label in this vulnerable patient group. A clear recommendation on the use of ACT could be difficult, due to a lack of data in infants with a body weight of less than $5 \mathrm{~kg}$. A more accurate estimate of the disease burden and parasite prevalence risk factors for infection including iron status, as well as the pharmacokinetic profile, safety and efficacy of currently available ACT in neonates from data provided by clinical trials underway will contribute to the development of the evidence-based treatment guidelines for this population, as well as aid the research and development of new drugs. It is definitely worth providing malaria control strategies that are aimed directly to this vulnerable age group, which will thereby to reduce neonatal morbidity and mortality

\section{References}

1. Steketee R, Wirima JJ, Slutsker L, Heymann DL, Breman JG (1996) The problem of malaria and malaria control in pregnancy in sub-Saharan Africa. Am J Trop Med Hyg 55: 2-7.

2. McDermott JM, Wirima JJ, Steketee RW, Breman JG, Heymann DL (1996) The effect of placental malaria infection on perinatal mortality in rural Malawi. Am J Trop Med Hyg 55: 61-65.

3. Tako EA, Zhou A, Lohoue J, Leke R, Taylor DW, et al. (2005) Risk factors for placental malaria and its effect on pregnancy outcome in Yaounde, Cameroon. Am J Trop Med Hyg 72: 236-242.

4. Ekanem AD, Anah MU, Udo JJ (2008) The prevalence of congenital malaria among neonates with suspected sepsis in Calabar, Nigeria. Trop Doct 38: 73-76

5. Falade C, Mokuolu O, Okafor H, Orogade A, Falade A, et al. (2007) Epidemiology of congenital malaria in Nigeria: a multi-centre study. Trop Med Int Health 12: 1279-1287.

6. Runsewe-Abiodun IT, Ogunfowora OB, Fetuga BM (2006) Neonatal malaria in Nigeria--a 2 year review. BMC Pediatr 6: 19.

7. Larru B, Molyneux E, Ter Kuile FO, Taylor T, Molyneux M, et al. (2009) Malaria in infants below six months of age: retrospective surveillance of hospital admission records in Blantyre, Malawi. Malar J 8: 310.

8. Akindele JA, Sowunmi A, Abohweyere AE (1993) Congenital malaria in a hyperendemic area: a preliminary study. Ann Trop Paediatr 13: 273-276.

9. Mukhtar MY, Lesi FE, Iroha EU, Egri-Okwaji MT, Mafe AG (2006) Congenital malaria among inborn babies at a tertiary centre in Lagos, Nigeria. J Trop Pediatr 52: 19-23.

10. Larkin GL, Thuma PE (1991) Congenital malaria in a hyperendemic area Am J Trop Med Hyg 45: 587-592.

11. Obiajunwa PO, Owa JA, Adeodu OO (2005) Prevalence of congenital malaria in lle-ife, Nigeria. J Trop Pediatr 51: 219-222.

12. Biggar RJ, Collins WE, Campbell CC (1980) The serological response to primary malaria infection in urban Ghanaian infants. Am J Trop Med Hyg 29: 720-724.

13. Sehgal VM, Siddjiqui WA, Alpers MP (1989) A seroepidemiological study to evaluate the role of passive maternal immunity to malaria in infants. Trans R Soc Trop Med Hyg 83 Suppl: 105-106. 
14. Brabin $B(1990)$ An analysis of malaria parasite rates in infants: 40 years after Macdonald. Trop Dis Bull: 87: 1-21.

15. Fischer PR (2003) Malaria and newborns. J Trop Pediatr 49: 132-134.

16. Afolabi BM, Salako LA, Mafe AG, Ovwigho UB, Rabiu KA, et al. (2001) Malaria in the first 6 months of life in urban African infants with anemia. Am J Trop Med Hyg 65: 822-827.

17. Riley EM, Wagner GE, Akanmori BD, Koram KA (2001) Do maternally acquired antibodies protect infants from malaria infection? Parasite Immunol 23: 51-59.

18. Riley EM, Wagner GE, Ofori MF, Wheeler JG, Akanmori BD, et al. (2000) Lack of association between maternal antibody and protection of African infants from malaria infection. Infect Immun 68: 5856-5863.

19. Doolan DL, Dobaño C, Baird JK (2009) Acquired immunity to malaria. Clin Microbiol Rev 22: 13-36.

20. Kassim OO, Ako-Anai KA, Torimiro SE, Hollowell GP, Okoye VC, et al. (2000) Inhibitory factors in breastmilk, maternal and infant sera against in vitro growth of Plasmodium falciparum malaria parasite. J Trop Pediatr 46: $92-96$.

21. Gitau GM, Eldred JM (2005) Malaria in pregnancy: clinical, therapeutic and prophylactic considerations. Obstet Gynaecol: 7:5-11.

22. Amaratunga C, Lopera-Mesa TM, Brittain NJ, Cholera R, Arie T, et al (2011) A role for fetal hemoglobin and maternal immune IgG in infant resistance to Plasmodium falciparum malaria. PLoS One 6: e14798.

23. Bardají A, Sigauque B, Sanz S, Maixenchs M, Ordi J, et al. (2011) Impact of malaria at the end of pregnancy on infant mortality and morbidity. J Infect Dis 203: 691-699.

24. Schwarz NG, Adegnika AA, Breitling LP, Gabor J, Agnandji ST, et al. (2008) Placental malaria increases malaria risk in the first 30 months of life. Clin Infect Dis 47: 1017-1025.

25. Malhotra I, Dent A, Mungai $P$, Wamachi A, Ouma JH, et al. (2009) Can prenatal malaria exposure produce an immune tolerant phenotype? A prospective birth cohort study in Kenya. PLoS Med 6: e1000116.

26. Olanrewaju WI (1999) Malaria in the neonate: report of 2 cases.West Afr J Med 18: 139-140.

27. Comellini L, Tozzola A, Baldi F, Brusa S, Serra L, et al. (1998) Plasmodium vivax congenital malaria in a newborn of a Zairian immigrant. Ann Trop Paediatr 18: 41-43.

28. Mukhtar M (2007) The growing incidence of neonatal malaria--a situational review in developing countries. Niger J Med 16: 25-30.

29. Fischer PR, Nyirjesy P, Toko RM (1995) Congenital malaria in twins. West J Med 163: 395-396.

30. Sacarlal J, Nhacolo AQ, Sigaúque B, Nhalungo DA, Abacassamo F, et al. (2009) A 10 year study of the cause of death in children under 15 years in Manhiça, Mozambique. BMC Public Health 9: 67.

31. World malaria report 2009, World Health Organization. WHO Press, Geneva, Switzerland

32. Tripathy R, Parida S, Das L, Mishra DP, Tripathy D, et al. (2007) Clinical manifestations and predictors of severe malaria in Indian children. Pediatrics 120: e454-460.

33. Hyacinth HI, Oguche S, Yilgwan CS (2012) Summary Description of 24 Cases of Neonatal Malaria Seen at a Tertiary Health Center in Nigeria. Iran J Pediatr 22: 87-91.

34. Lehner PJ, Andrews CJ (1988) Congenital malaria in Papua New Guinea. Trans R Soc Trop Med Hyg 82: 822-826.

35. Quinn TC, Jacobs RF, Mertz GJ, Hook EW 3rd, Locklsey RM (1982) Congenital malaria: a report of four cases and a review. J Pediatr 101 229-232

36. Singh J, Soni D, Mishra D, Singh HP, Bijesh S (2014) Placental and neonatal outcome in maternal malaria. Indian Pediatr 51: 285-288.

37. Runsewe-Abiodun IT, Ogunfowora OB, Fetuga BM (2006) Neonatal malaria in Nigeria--a 2 year review. BMC Pediatr 6: 19

38. Corkill JA, Brabin BJ, MacGregor DF, Alpers MP, Milner RD (1989)
Newborn splenic volumes vary under different malaria endemic conditions Arch Dis Child 64: 541-545.

39. Reyburn H, Mbatia R, Drakeley C, Carneiro I, Mwakasungula E, et al (2004) Overdiagnosis of malaria in patients with severe febrile illness in Tanzania: a prospective study. BMJ 329: 1212

40. Zurovac D, Midia B, Ochola SA, English M, Snow RW (2006) Microscopy and outpatient malaria case management among older children and adults in Kenya. Trop Med Int Health 11: 432-440.

41. Makani J, Matuja W, Liyombo E, Snow RW, Marsh K, et al. (2003) Admission diagnosis of cerebral malaria in adults in an endemic area of Tanzania: implications and clinical description. QJM 96: 355-362.

42. Mwaniki MK, Talbert AW, Mturi FN, Berkley JA, Kager P, et al. (2010) Congenital and neonatal malaria in a rural Kenyan district hospital: an eight-year analysis. Malar J 9: 313.

43. Gurrant RL, Walker DH, Weller PF, Trapil (1999) Infection disease principles Pathogens \& practice churchill livingstone 1: 736-166.

44. Behrman RE, Keligman R, Jenson HB (2011) Nelson Text book of Pediatrics. Philadelphia: Sounders 19: 1201

45. Singh N, Shukla MM, Sharma VP (1999) Epidemiology of malaria in pregnancy in central India. Bull World Health Organ 77: 567-572.

46. COVELL G (1950) Congenital malaria. Trop Dis Bull 47: 1147-1167.

47. Patwari A, Aneja S, Berry AM, Ghosh S (1978) Neonatal malaria--a clinico-hematological profile. Indian Pediatr 15: 847-849.

48. Choudhary SP, Dabi DR, Singh RN, Theraja SL (1983) Malaria in newborn. Indian Pediatr 20: 41-43.

49. Singh T (1985) Neonatal malaria. Indian Pediatr 22: 785-786.

50. Bruce-Chwatt LJ (1970) Acute malaria in newborn infants. Br Med Jour 3: 283.

51. Thapa BR, Narang A, Bhakoo ON (1987) Neonatal malaria: a clinical study of congenital and transfusional malaria. J Trop Pediatr 33: 266-268.

52. Piñeros-Jiménez JG, Álvarez G, Tobón A, Arboleda M, Carrero S, et al (2011) Congenital malaria in Urabá, Colombia. Malar J 10: 239.

53. Steketee RW, Wirima JJ, Slutsker L, Roberts JM, Khoromana CO, et al. (1996) Malaria parasite infection during pregnancy and at delivery in mother, placenta, and newborn: efficacy of chloroquine and mefloquine in rural Malawi. Am J Trop Med Hyg 55: 24-32.

54. Singh M (2010) Congenital malaria care of the newborn, Sagar printers \& publishers 16: 211

55. Fischer PR (1997) Congenital malaria: an African survey. Clin Pediatr (Phila) 36: 411-413

56. Kamwendo DD, Dzinjalamala FK, Snounou G, Kanjala MC, Mhango CG et al. (2002) Plasmodium falciparum: PCR detection and genotyping of isolates from peripheral, placental, and cord blood of pregnant Malawian women and their infants. Trans R Soc Trop Med Hyg 96: 145-149.

57. Uneke CJ (2007) Congenital Plasmodium falciparum malaria in subSaharan Africa: a rarity or frequent occurrence? Parasitol Res 101: 835-842.

58. Mohan K, Maithani MM (2010) Congenital malaria due to chloroquineresistant Plasmodium vivax: a case report. J Trop Pediatr 56: 454-455.

59. Adachi M, Manji K, Ichimi R, Nishimori H, Shindo K, et al. (2000) Detection of congenital malaria by polymerase-chain-reaction methodology in Dar es Salaam, Tanzania. Parasitol Res 86: 615-618.

60. Hailegiorgis B, et al (2010): Laboratory malaria diagnostic capacity in health facilities in five administrative zones of oromia regional state. Ethiopia. Trop Med Int Health, 15:1449-1457.

61. Ishengoma DR, Derua YA, Rwegoshora RT, Tenu F, Massaga JJ, et al. (2010) The performance of health laboratories and the quality of malaria diagnosis in six districts of Tanzania. Ann Trop Med Parasitol 104: $123-135$.

62. Lawn JE, Cousens S, Zupan J; Lancet Neonatal Survival Steering Team (2005) 4 million neonatal deaths: when? Where? Why? Lancet 365 $891-900$. 
Citation: Mohan K, Omar BJ, Singh RD (2014) Clinical Presentation and Management of Neonatal Malaria: A Review. Malar Chemoth Cont Elimination 3: 126. doi: 10.4172/2090-2778.1000126

Page 5 of 2

63. Abdullah S, Adazu K, Masanja H, Diallo D, Hodgson A, et al. (2007) Patterns of age-specific mortality in children in endemic areas of subSaharan Africa. Am J Trop Med Hyg 77: 99-105.

64. Sacarlal J, Nhacolo AQ, Sigaúque B, Nhalungo DA, Abacassamo F, et al. (2009) A 10 year study of the cause of death in children under 15 years in Manhiça, Mozambique. BMC Public Health 9: 67.

65. Kearns GL, Abdel-Rahman SM, Alander SW, Blowey DL, Leeder JS et al. (2003) Developmental pharmacology--drug disposition, action, and therapy in infants and children. N Engl J Med 349: 1157-1167.

66. WHO (2010) Guidelines for the treatment of Malaria. Geneva, Switzerland: World Health Organization.

67. Milsap RL, Jusko WJ (1994) Pharmacokinetics in the infant. Environ Health Perspect 102 Suppl 11: 107-110.
68. Menendez C, Mayor A (2007) Congenital malaria: the least known consequence of malaria in pregnancy. Semin Fetal Neonatal Med 12: 207-213.

69. Orogadeav A A et al (2008): Clinical and laboratory features in congenital malaria in Nigeria. J. Pediatr Infec Dis 3: 181-187

70. WHO (2004) A Strategic Framework for malaria prevention and control during pregnancy in the African Region. Brazzaville: World Health Organization Regional Office for Africa.

71. Federal Ministry of Health. National Guidelines and Strategies for Prevention and Control of malaria during Pregnancy. Abuja, Federal Government of Nigeria. 